\title{
Particle Acceleration by an Electromagnetic Wave
}

\author{
Heinz Dehnen \\ Fachbereich Physik, Universität Konstanz, Postfach 677, 78457 Konstanz, Germany \\ Correspondence should be addressed to Heinz Dehnen; heinz.dehnen@uni-konstanz.de
}

Received 3 July 2013; Accepted 6 August 2013

Academic Editors: M. Alishahiha, F. Brito, and K. Cho

Copyright (C) 2013 Heinz Dehnen. This is an open access article distributed under the Creative Commons Attribution License, which permits unrestricted use, distribution, and reproduction in any medium, provided the original work is properly cited.

The relativistic equation of motion for an electrically charged massive point particle is integrated exactly for the case of a circularly polarized electromagnetic plane wave. The acceleration of the particle by the wave is calculated in detail, however, under neglection of the radiation damping. In spite of this simplification, the results are applied to a typical pulsar, where the maximum kinetic energy of accelerated protons reaches values of the order of the fastest cosmic ray particles.

\section{Introduction}

The present model for a pulsar is that of an oblique rotator, where the magnetic moment $\vec{M}$ of the neutron star is inclined against the rotation axis of the star ( $z$-axis) by an angle $\theta$. Then, in consequence of the time variable magnetic moment $\vec{M}_{\perp}$ perpendicular to the rotation axis, a magnetic dipole radiation is emitted, which is circularly polarized (see Figure 1).

For this reason it may be interesting to calculate the acceleration of an electrically charged particle by a circularly polarized electromagnetic wave propagating in the $z$ direction. For simplicity, we assume a plane wave with the field strength

$$
\begin{gathered}
E_{x}=E_{0} \cos (\omega t-k z), \\
E_{y}=E_{0} \sin (\omega t-k z), \\
E_{z}=0, \\
B_{x}=-E_{0} \sin (\omega t-k z), \\
B_{y}=E_{0} \cos (\omega t-k z), \\
B_{z}=0
\end{gathered}
$$

satisfying the sourceless Maxwell equations. An additional constant phase parameter is not necessary in (1), because the $x$ - and $y$-axis can be chosen in any case in such a way that (1) is valid by turning around the $z$-axis. (This simplification is not possible in the case of a linearly polarized wave.) The application of the ansatz (1) to a neutron star is possible only with reservation, first because the electromagnetic wave field (1) represents only the electromagnetic far field and second because the decrease of the intensity of the wave with increasing distance from the star is not taken into account. However, the maximum of the particle energy attainable by such an acceleration process may be evaluable, which is of interest for explaining the energy observed in cosmic rays. Beyond this, the results may be applicable in the accelerator physics.

Such calculations are already performed for pulsars first by Gunn and Ostriker [1,2] in 1969 as well as later by Grewing and Heintzmann [3] and for accelerators by Holkundkar et al. [4]. But all these calculations are not performed by an exact analytical integration of the relativistic equation of motion of the charged particle. To do that is the aim of the present paper.

\section{Solution of the Equation of Motion}

The relativistic equation of motion

$$
\frac{d}{d t}\left(\frac{m_{0} \vec{v}}{\sqrt{1-\beta^{2}}}\right)=e \vec{E}+\frac{e}{c} \vec{v} \times \vec{B}, \quad \beta=\frac{|\vec{v}|}{c}
$$




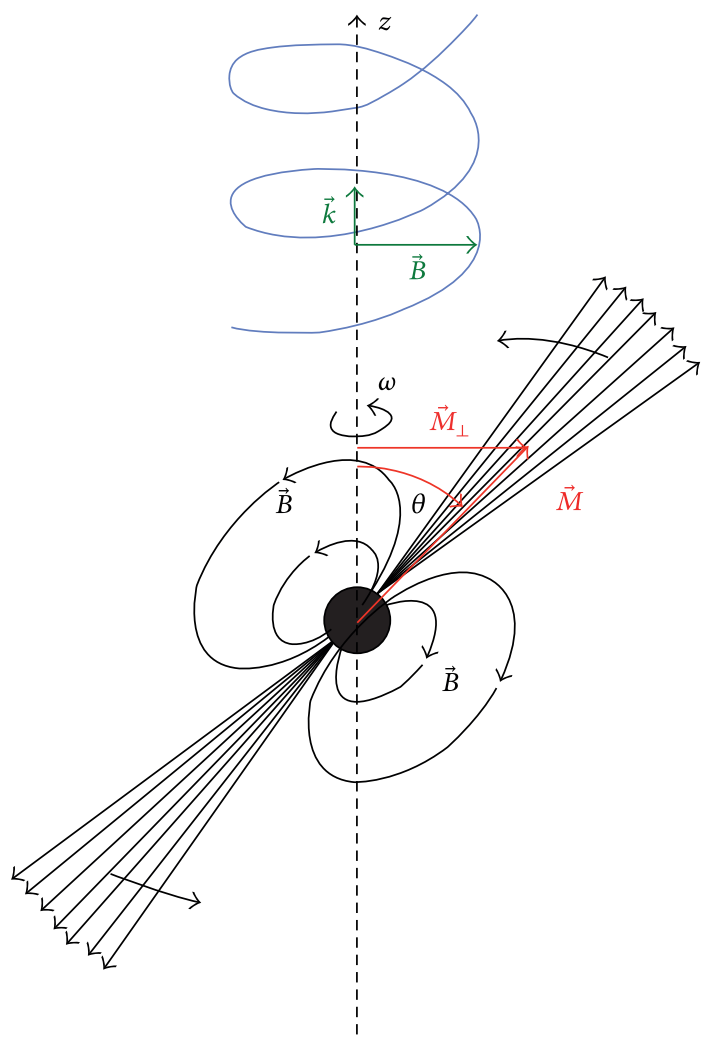

FIGURE 1: Oblique rotator as pulsar model.

for a charged point particle (rest mass $m_{0}$, electric charge $e$ ) reads in the case of the field (1) in cartesian coordinates

$$
\begin{gathered}
\frac{d}{d t}\left(\frac{m_{0} v_{x}}{\sqrt{1-\beta^{2}}}\right)=e E_{x}-\frac{e}{c} v_{z} B_{y} \\
\frac{d}{d t}\left(\frac{m_{0} v_{y}}{\sqrt{1-\beta^{2}}}\right)=e E_{y}+\frac{e}{c} v_{z} B_{x} \\
\frac{d}{d t}\left(\frac{m_{0} v_{z}}{\sqrt{1-\beta^{2}}}\right)=\frac{e}{c}\left(v_{x} B_{y}-v_{y} B_{x}\right) .
\end{gathered}
$$

The radiation damping is neglected for simplicity. Accordingly, a particle at rest will be accelerated at first by the electric field in $x$ - and $y$-direction and then by the magnetic field in $z$-direction. Furthermore, from (2) the energy law follows immediately:

$$
\frac{d}{d t}\left(\frac{m_{0} c^{2}}{\sqrt{1-\beta^{2}}}\right)=e \vec{v} \vec{E}
$$

which takes the following form according to (1):

$$
\frac{d}{d t}\left(\frac{m_{0} c^{2}}{\sqrt{1-\beta^{2}}}\right)=e\left(v_{x} E_{x}+v_{y} E_{y}\right) .
$$

Insertion of (1) into the first two equations of (3) gives $(\cdot \hat{=} d / d t)$

$$
\begin{aligned}
& \frac{d}{d t}\left(\frac{m_{0} \dot{x}}{\sqrt{1-\beta^{2}}}\right)=e E_{0}\left(1-\frac{\dot{z}}{c}\right) \cos (\omega t-k z), \\
& \frac{d}{d t}\left(\frac{m_{0} \dot{y}}{\sqrt{1-\beta^{2}}}\right)=e E_{0}\left(1-\frac{\dot{z}}{c}\right) \sin (\omega t-k z) .
\end{aligned}
$$

Because of the dispersion relation

$$
\frac{\omega}{k}=c
$$

of the electromagnetic wave, the integrals of (6) read

$$
\begin{gathered}
\frac{m_{0} \dot{x}}{\sqrt{1-\beta^{2}}}=\frac{e E_{0}}{\omega} \sin (\omega t-k z) \quad(\gtrless 0), \\
\frac{m_{0} \dot{y}}{\sqrt{1-\beta^{2}}}=\frac{e E_{0}}{\omega}[1-\cos (\omega t-k z)] \quad(\geqslant 0),
\end{gathered}
$$

using the following initial condition ( $z=0$ may be identified later with the surface of the star):

$$
t=0: \quad x=y=z=0 ; \quad \dot{x}=\dot{y}=\dot{z}=0,
$$

which means acceleration out of the rest state. Herewith, the third equation of (3) and (5) take the following form using (1):

$$
\begin{aligned}
& \frac{d}{d t}\left(\frac{m_{0} \dot{z}}{\sqrt{1-\beta^{2}}}\right)=\frac{e^{2} E_{0}^{2}}{m_{0} c \omega} \sqrt{1-\beta^{2}} \sin (\omega t-k z), \\
& \frac{d}{d t}\left(\frac{m_{0} c^{2}}{\sqrt{1-\beta^{2}}}\right)=\frac{e^{2} E_{0}^{2}}{m_{0} \omega} \sqrt{1-\beta^{2}} \sin (\omega t-k z) .
\end{aligned}
$$

Comparison of these two equations gives

$$
\frac{d}{d t} \frac{m_{0} \dot{z}}{\sqrt{1-\beta^{2}}}=\frac{d}{d t} \frac{m_{0} c}{\sqrt{1-\beta^{2}}}
$$

with the integral taking into account the initial values (9)

$$
\frac{m_{0} \dot{z}}{\sqrt{1-\beta^{2}}}=m_{0} c\left(\frac{1}{\sqrt{1-\beta^{2}}}-1\right) \text {. }
$$


By squaring of (8) and (12) and by addition, we find the following kinetic energy:

$$
T=m_{0} c^{2}\left(\frac{1}{\sqrt{1-\beta^{2}}}-1\right)=2 \frac{e^{2} E_{0}^{2}}{m_{0} \omega^{2}} \sin ^{2} \frac{1}{2}(\omega t-k z),
$$

and herewith from (12) analogously to (8) we have

$$
\frac{m_{0} \dot{z}}{\sqrt{1-\beta^{2}}}=2 \frac{e^{2} E_{0}^{2}}{m_{0} c \omega^{2}} \sin ^{2} \frac{1}{2}(\omega t-k z) \text {. }
$$

The substitution of the square root in (14) by the use of (13) results finally in

$$
\begin{array}{r}
\dot{z}=\frac{\left(2 e^{2} E_{0}^{2} / m_{0}^{2} c \omega^{2}\right) \sin ^{2}(1 / 2)(\omega t-k z)}{1+\left(2 e^{2} E_{0}^{2} / m_{0}^{2} c^{2} \omega^{2}\right) \sin ^{2}(1 / 2)(\omega t-k z)} \\
(\dot{z} \geq 0)
\end{array}
$$

and from (8) and (14), one finds the following quotient:

$$
\frac{\dot{z}}{\dot{y}}=\frac{e E_{0}}{m_{0} c \omega} .
$$

For the acceleration in $z$-direction, one obtains from (15) immediately

$$
\ddot{z}=\frac{e^{2} E_{0}^{2}}{m_{0}^{2} c \omega} \frac{\sin (\omega t-k z)}{\left[1+2\left(e^{2} E_{0}^{2} / m_{0}^{2} c^{2} \omega^{2}\right) \sin ^{2}(1 / 2)(\omega t-k z)\right]^{3}} .
$$

The maximum velocity $\dot{z}$ will be reached for $\omega t-k z=\pi$ and amounts to

$$
\frac{\dot{z}_{\max }}{c}=\frac{2\left(e^{2} E_{0}^{2} / m_{0}^{2} c^{2} \omega^{2}\right)}{\left(1+2\left(e^{2} E_{0}^{2} / m_{0}^{2} c^{2} \omega^{2}\right)\right)}
$$

For $e E_{0} / m_{0} c \omega \gg 1$, it is valid: $\dot{z}_{\max } \rightarrow c$.

Obviously, the maximum kinetic energy $T$, which can be reached by the acceleration of the electromagnetic wave, is given according to (13) also for $\omega t-k z=\pi$ by

$$
T_{\max }=2 \frac{e^{2} E_{0}^{2}}{m_{0} \omega^{2}} .
$$

Evidently, the energy transfer is very effective for low frequencies and high intensities. However, the intensities are correlated with at least the fourth power of the frequencies so that pulsars with high frequencies will produce the particles with the highest energies; see Section 3.

Finally, the total integration of the equations of motion is possible; however, of especial interest is the motion in $z$ direction. With the substitution

$$
\phi=\omega t-k z
$$

one obtains from (12) and (15)

$$
\begin{aligned}
\dot{\phi} & =\frac{\omega}{1+\left(e^{2} E_{0}^{2} / m_{0}^{2} c^{2}\right)\left(1 / \omega^{2}\right) \sin ^{2}(1 / 2) \phi} \\
& =\omega \sqrt{1-\beta^{2}}
\end{aligned}
$$

with the integral for $\phi(t)$ being valid for the moving particle

$$
\frac{e^{2} E_{0}^{2}}{m_{0}^{2} c^{2} \omega^{2}}(\phi-\sin \phi)+\phi=\omega t
$$

using the initial values of (9). Subsequently, it follows for $z(t)$ from (19):

$$
z(t)=c t-\frac{c}{\omega} \phi(t) .
$$

The acceleration of the particle in positive $z$-direction happens according to (17), see also (18), within the range

$$
0 \leq \phi \leq \pi \text {. }
$$

Then, the end of the acceleration phase $(\phi=\pi)$ is reached according to (21) for

$$
t_{\max }=\left(\frac{e^{2} E_{0}^{2}}{m_{0}^{2} c^{2} \omega^{3}}+\frac{1}{\omega}\right) \pi
$$

at (see (22))

$$
z_{\max }=\pi \frac{e^{2} E_{0}^{2}}{m_{0}^{2} c \omega^{3}} .
$$

If $z_{\max }$ is very large, the intensity of a real spherical wave is decreased drastically so that the interaction between particle and wave can be considered as finished. Otherwise the particle will be decelerated and reaches the initial velocity $\dot{z}=0$ according to (15) for $\phi=2 \pi$ corresponding to $\omega t=$ $2 \pi\left(1+\left(e^{2} E_{0}^{2} / m_{0}^{2} c^{2} \omega^{2}\right)\right)$ and $z=2 \pi\left(e^{2} E_{0}^{2} / m_{0}^{2} c \omega^{3}\right)$.

For completeness, we integrate also the motion in $x$ - and $y$-direction. Equation (20) gives

$$
\sqrt{1-\beta^{2}}=\frac{\dot{\phi}}{\omega} \text {. }
$$

Then, it follows from (8) that

$$
m \dot{x}=\frac{e E_{0}}{\omega^{2}} \dot{\phi} \sin \phi, \quad m_{0} \dot{y}=\frac{e E_{0}}{\omega^{2}} \dot{\phi}(1-\cos \phi),
$$

with the integrals using the initial condition (9)

$$
x=\frac{e E_{0}}{m_{0} \omega^{2}}(1-\cos \phi), \quad y=\frac{e E_{0}}{m_{0} \omega^{2}}(\phi-\sin \phi),
$$

where $\phi(t)$ is given by (21). It is interesting that the very easy integration is possible because of the relativistic factor (26) in (8) (velocity dependence of the mass). Obviously, $\dot{x}=0$ is valid for $\phi=\pi$, where $\dot{x}$ changes its sign and $x$ goes back to zero $(x=0)$ for $\phi=2 \pi$. In contrast to this, $\dot{y}$ reaches its maximum for $\phi=\pi$ with the value $\dot{y}=c\left(e E_{0} / \omega c m_{0}\right)(2 /(1+$ $\left.\left.2\left(e^{2} E_{0}^{2} / m_{0}^{2} c^{2} \omega^{2}\right)\right)\right)$, and $\dot{y}=0$ is valid for $\phi=2 \pi$, where $y=2 \pi\left(e E_{0} / m_{0} \omega^{2}\right)$. For $\left(e E_{0} / m_{0} c \omega\right) \gg 1$, the maximum $y$ velocity is small compared with $c$ and $z(2 \pi) / y(2 \pi) \gg 1$ is valid so that the acceleration happens mainly in the direction of the wave ( $z$-direction). 


\section{Application to a Neutron Star}

For the comparison with a real situation, we choose a neutron star with the following data ( $R$ radius of the star, $v$ rotating frequency):

$$
\begin{gathered}
R=10 \mathrm{~km}, \quad v=100 \mathrm{~Hz}, \\
\left|\vec{B}_{\text {pole }}\right|=10^{12} \text { Gauß. }
\end{gathered}
$$

For the dipole radiation intensity $\vec{S}$ (Poynting vector), it is valid:

$$
|\vec{S}|=\frac{\ddot{\vec{M}}_{\perp}^{2}}{4 \pi c^{3} r^{2}} \sin ^{2} \chi,
$$

where $\chi$ is the angle between $\vec{S}$ and the magnetic moment $\vec{M}_{\perp}$. For radiation in the direction of the rotation axis ( $z$-axis) of the pulsar, $\chi=\pi / 2$ holds (maximum intensity). On the other hand, we use for the connection between the magnetic field strength $\vec{B}$ and the magnetic moment $\vec{M}$ the relation for a barmagnet

$$
B_{z^{\prime}}=-\frac{M_{z^{\prime}}}{r^{3}}\left(1-3 \frac{z^{\prime 2}}{r^{2}}\right)
$$

outside, but on the axis ( $z^{\prime}$-axis) of the magnet. At the magnetic pole $\left(z^{\prime}=r=R\right)$ we find

$$
\vec{B}_{\text {pole }}=2 \frac{\vec{M}}{R^{3}}
$$

Furthermore, for the oblique rotator it is valid ( $\theta$ angle between $\vec{M}$ and $z$-axis (see Figure 1) as follows:

$$
\left|\vec{M}_{\perp}\right|=|\vec{M}| \sin \theta \text {, }
$$

where (cf. (1) and (32)) $\vec{M}_{\perp}=\left|\vec{M}_{\perp}\right|(-\sin \omega t, \cos \omega t, 0)$. (Vector in the $x ; y$-plane perpendicular to the rotation axis (z-axis).) Herewith and with $|\vec{S}|=(c / 4 \pi) \vec{E}^{2}$, we find from (30) for the wave intensity the following

$$
\vec{E}^{2}=E_{0}^{2}=\frac{\omega^{4}}{c^{4} R^{2}} \vec{M}_{\perp}^{2}
$$

at the rotation pole of the star $(r=R)$. Insertion of (32) into (33) and of (33) into (34) results in

$$
E_{0}^{2}=\frac{1}{4} \frac{\vec{B}_{\text {pole }}^{2} R^{4} \omega^{4}}{c^{4}} \sin ^{2} \theta .
$$

Herewith, we find from (18) the maximum kinetic energy (the total radiation power of the dipole-radiation of the pulsar amounts to $9,64 \cdot 10^{38} \sin ^{2} \theta \mathrm{erg} / \mathrm{sec}$ ) as follows:

$$
\begin{aligned}
T_{\max } & =\frac{e^{2} \vec{B}_{\text {pole }}^{2} R^{4} \omega^{2}}{2 m_{0} c^{4}} \sin ^{2} \theta \\
& =3,4 \cdot 10^{16} \sin ^{2} \theta \mathrm{erg} \\
& =2,1 \cdot 10^{28} \sin ^{2} \theta \mathrm{eV}
\end{aligned}
$$

for protons as particles and the star data (29). For the acceleration time (24), one obtains in the case of protons

$$
\begin{aligned}
t_{\max } & =\frac{\pi}{\omega}\left[\frac{1}{4} \frac{e^{2} \vec{B}_{\text {pole }}^{2} R^{4} \omega^{2}}{m_{0}^{2} c^{6}} \sin ^{2} \theta+1\right] \\
& =5,64 \cdot 10^{16} \sin ^{2} \theta \mathrm{sec} \\
& =1,79 \cdot 10^{9} \sin ^{2} \theta \mathrm{y},
\end{aligned}
$$

and for the acceleration distance (25) one obtains

$$
\begin{aligned}
z_{\max } & =\frac{\pi}{4} \frac{e^{2} \vec{B}_{\text {pole }}^{2} R^{4} \omega}{m_{0}^{2} c^{5}} \sin ^{2} \theta \\
& =1,69 \cdot 10^{27} \sin ^{2} \theta \mathrm{cm} \\
& =1,79 \cdot 10^{9} \sin ^{2} \theta \mathrm{ly} .
\end{aligned}
$$

Obviously, the path length of the light $c t_{\max }$ is larger than $z_{\max }$ only by $\lambda / 2$ of the wave as follows:

$$
c t_{\max }-z_{\max }=\frac{c \pi}{\omega}=\frac{\lambda}{2} \quad\left(\lambda=3 \cdot 10^{8} \mathrm{~cm}\right) .
$$

Otherwise, the negative half-wave will be important and the particle will be retarded, cf. (17). However, because of $z_{\max } \gg$ $\lambda / 2$, the relation (39) means that the particle runs nearly with the speed of light surfriding on the wave. For the ratio (16) of the velocities in $z$-and $y$-direction one gets

$$
\frac{\dot{z}}{\dot{y}}=\frac{1}{2} \frac{e\left|\vec{B}_{\text {pole }}\right| R^{2} \omega}{m_{0} c^{3}} \sin \theta=3,3 \cdot 10^{9} \sin \theta,
$$

so that the motion results mostly in the $z$-direction, that is, in the direction of the wave. Finally, we see that also very small angles, for example, $\sin ^{2} \theta=10^{-7}$ result in reasonable values for $T_{\max }, t_{\max }$, and $z_{\max }$ for protons:

$$
T_{\max }=2 \cdot 10^{21} \mathrm{eV}, \quad t_{\max }=180 \mathrm{y}, \quad z_{\max } \lesssim 180 \mathrm{ly} .
$$

At the end of the acceleration phase $(\theta=\pi)$ it follows:

$$
\begin{aligned}
x_{\text {max }} & =\frac{e\left|B_{\text {pole }}\right| R^{2}}{m_{0} c^{2}} \sin \theta \\
& =3,2 \cdot 10^{17} \sin \theta \mathrm{cm} \\
& =0,3 \sin \theta \mathrm{ly}, \\
y_{\max } & =\frac{\pi}{2} \frac{e\left|B_{\text {pole }}\right| R^{2}}{m_{0} c^{2}} \sin \theta \\
& =4,9 \cdot 10^{17} \sin \theta \mathrm{cm} \\
& =0,5 \sin \theta \mathrm{ly} .
\end{aligned}
$$

The comparison with $z_{\max }$, (38), shows once more that the accelerated motion results mostly in the $z$-direction. 
TABLE 1: Attainable energies $T$ for protons in dependence of the phase $\phi$ and of the distance $z$ from the pulsar.

\begin{tabular}{lcccccc}
\hline$\phi$ & $10^{-6}$ & $10^{-5}$ & $10^{-4}$ & $10^{-3}$ & $10^{-2}$ & \\
$z / \sin ^{2} \theta$ & $8,9 \cdot 10^{2}$ & $8,9 \cdot 10^{5}$ & $8,9 \cdot 10^{8}$ & $8,9 \cdot 10^{11}$ & $8,9 \cdot 10^{14}$ & $\mathrm{~km}$ \\
$T / \sin ^{2} \theta$ & $5,25 \cdot 10^{15}$ & $5,25 \cdot 10^{17}$ & $5,25 \cdot 10^{19}$ & $5,25 \cdot 10^{21}$ & $5,25 \cdot 10^{23}$ & $\mathrm{eV}$ \\
\hline
\end{tabular}

Besides these maximum values, it is of especial interest to determine the attainable energy $T$ in the distance $z$ from the star in dependence of the phase $\phi$. From (21) to (22), and from (13) to (19) it follows immediately:

$$
\begin{gathered}
z=\frac{e^{2} E_{0}^{2}}{m_{0}^{2} c \omega^{3}}(\phi-\sin \phi), \\
T=2 \frac{e^{2} E_{0}^{2}}{m_{0} \omega^{2}} \sin ^{2} \frac{\phi}{2} .
\end{gathered}
$$

In Table 1, we show the values for $z$ and $T$ in the range $0<$ $\phi<10^{-2}$ for protons and the neutron star data (29) (see also (35)). It is interesting that up to distances of $10^{15} \mathrm{~km}$ $\phi \ll 1$ is valid, which demonstrates once more that the protons surfride on the wave very early, so that $\phi$ changes its values only very slowly. On the other hand, the large $z$ values for higher energies show clearly that the neglection of the decrease of the wave intensity with increasing distance is indeed a very rough approximation.

\section{Final Remark}

Some critical remarks are necessary concerning the application of the results of Section 2 to neutron stars. The reached maximum energy of protons is indeed of the order of magnitude of cosmic rays. However, we have neglected the enormous gravitational attraction of the particle by the neutron star. Furthermore, we have used the following simplifications:

(1) plane wave instead of a spherical one,

(2) using only the radiation far field,

(3) neglection of the influences on the wave by the surrounding plasma (see e.g., Kegel [5] and Thielheim [6]) and by the strong gravitational field,

(4) neglection of the radiation damping.

Therefore, the results for the neutron star can be considered only as very rough estimations.

\section{References}

[1] J. E. Gunn and J. P. Ostriker, "Acceleration of high-energy cosmic rays by pulsars," Physical Review Letters, vol. 22, no. 14, pp. 728-731, 1969.

[2] J. E. Gunn and J. P. Ostriker, "On the nature of pulsars. I. Theory," The Astrophysical Journal, vol. 157, pp. 1395-1417, 1969.

[3] M. Grewing and H. Heintzmann, "On the acceleration of charged particles to cosmic ray energies," Astronomy Letters, vol. 8, p. $167,1971$.
[4] A. Holkundkar, G. Brodin, and M. Marklund, "Proton acceleration by circularly polarized traveling electromagnetic wave," Physical Review Special Topics, vol. 15, Article ID 091301, 2012.

[5] W. H. Kegel, "On the acceleration of cosmic ray particles by pulsar fields," Astronomy \& Astrophysics, vol. 12, p. 452, 1971.

[6] K. O. Thielheim, "Particle dynamics in spherical wave fields of rotating magnets: acceleration boundary, plasma border, and 1/r02 law of asymptotic energy," Astrophysical Journal Letters, vol. 409, no. 1, pp. 333-344, 1993. 

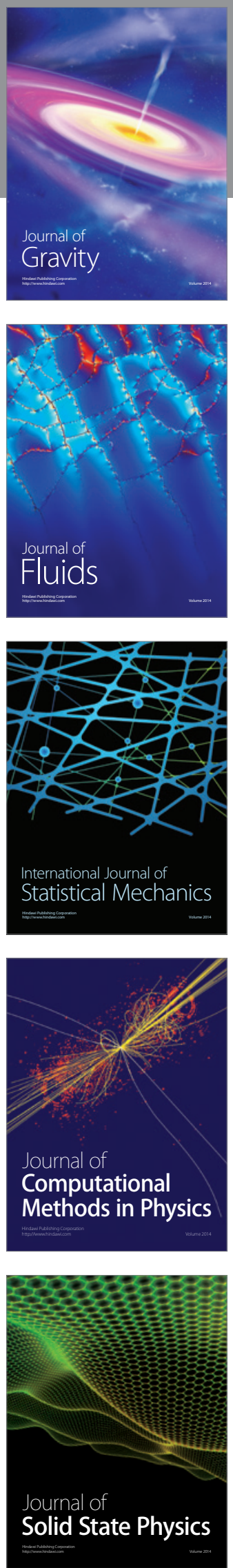

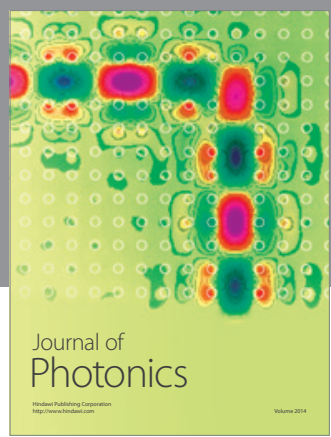

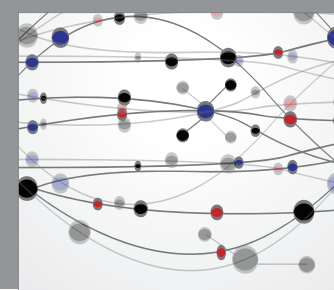

The Scientific World Journal

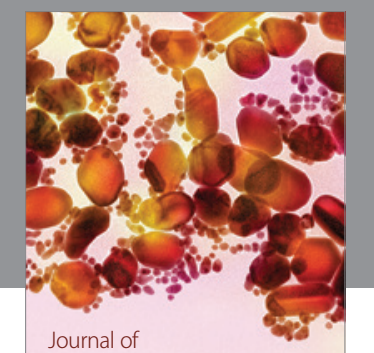

Soft Matter
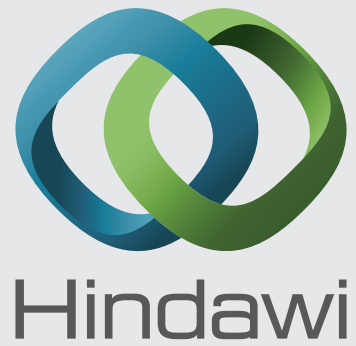

Submit your manuscripts at

http://www.hindawi.com
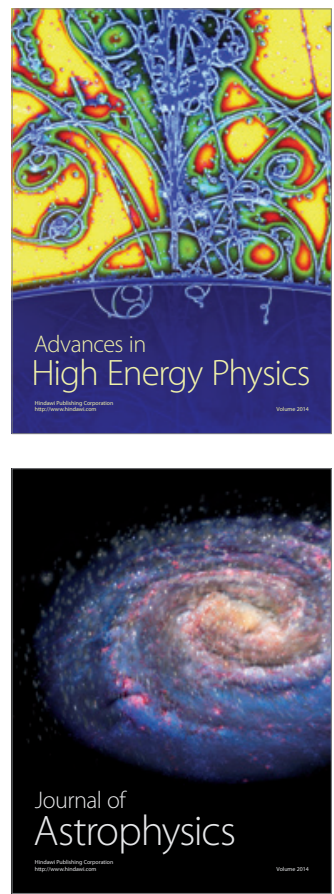
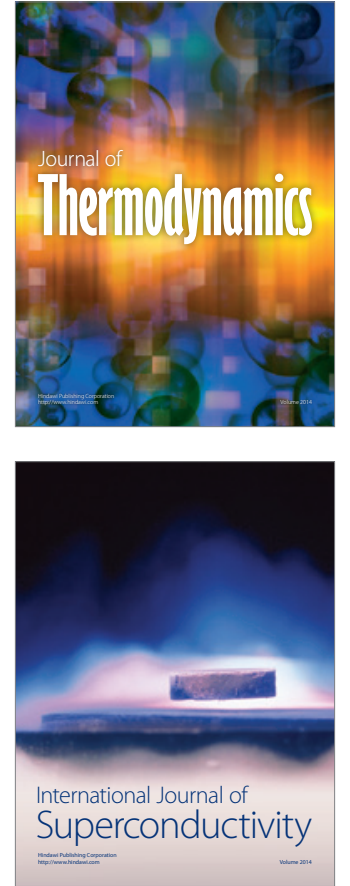
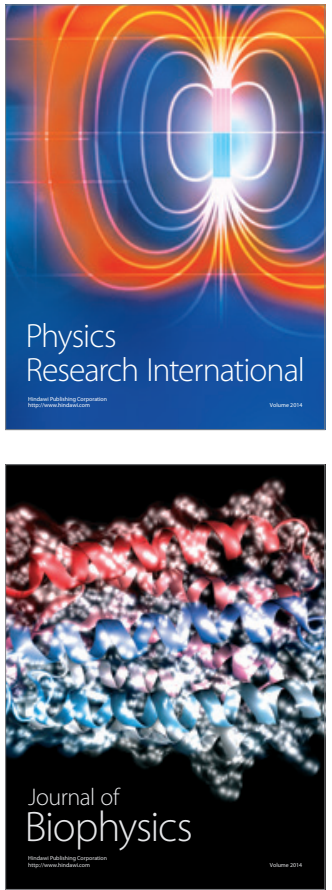
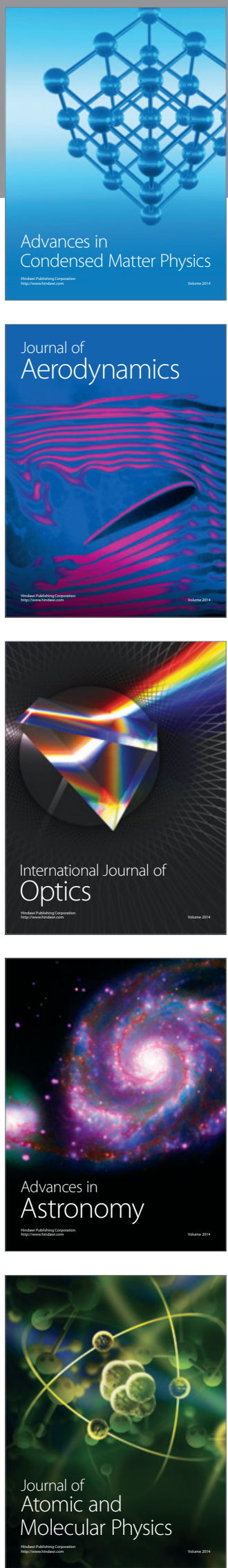